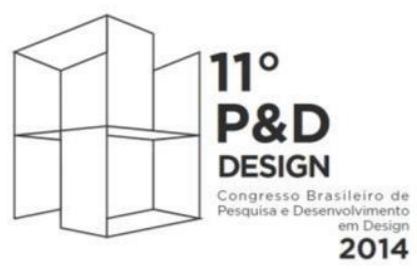

Gramado - RS

De 30 de setembro a 2 de outubro de 2014

\title{
ESTUDO SOBRE DIMENSÃO DE ESTAMPA LOCALIZADA EM ARTIGO DE VESTUÁRIO PERSONALIZADO: adequação de estampa a partir da antropometria e da digitalização tridimensional do corpo humano
}

\author{
Bruna Barth Bertotto \\ Universidade Federal do Rio Grande do Sul \\ brunabbertotto@gmail.com \\ Nathalia Alborghetti Carvalho \\ Universidade Federal do Rio Grande do Sul \\ nathaliaalc@yahoo.com.br \\ Fábio Pinto da Silva \\ Universidade Federal do Rio Grande do Sul \\ fabio.silva@ufrgs.br
}

\begin{abstract}
Resumo: Considerando os avanços das tecnologias tridimensionais e dos estudos antropométricos, a proposta deste estudo é analisar o tamanho mais favorável de estampas localizadas através da digitalização 3D, baseadas nos percentis de estatura. Pode-se dizer que o aprimoramento da antropometria se deu através dos sistemas de digitalização do corpo humano, que existem a mais de quinze anos. O objetivo da pesquisa é verificar a viabilidade e eficiência do escaneamento do corpo humano como ferramenta de apoio à estamparia localizada para a aplicação de estampagem, tendo em vista que um dos maiores negócios do mercado é a indústria de moda. Fato que se deve não só ao alto volume de vendas e a sucessão de tendências em curto espaço de tempo, mais também à quantidade de pessoas e empresas envolvidas desde a concepção dos produtos até sua distribuição. Essa é uma das causas de interesse em utilizar essas tecnologias à moda e indústria do vestuário. Quanto mais rápidos e precisos forem os estudos de modelagem, estética e design dos produtos, mais competitivos esses eles se tornam. Com fins comparativos os testes foram realizados com três modelos masculinos e três modelos femininos enquadrados em três percentis de estatura (5, 50 e $95 \%)$ com idades entre 18 e 34 anos, onde se aplicou a mesma estampa localizada nos seis modelos, comparando o comportamento da mesma em cada um.
\end{abstract}




\title{
Palavras-chave: Digitalização tridimensional, Antropometria, Design de superfície.
}

\begin{abstract}
Considering three-dimensional technologies and advances of anthropometric studies, the aim of this study is to assess the size and the more favourable positioning of pictures located through 3D scanning, based on percentiles of stature. It can be said that the improvement of the anthropometry through scanning systems of the human body, there are more than fifteen years. The purpose of the research is to ascertain the viability and efficiency of the human body scanning for the stamping application, considering that one of the biggest businesses in the market is the Fashion industry. Fact that one must not only to the high volume of sales and the succession of trends in short time, but also the amount of people and companies involved from the conception of the products until delivery. This is one of the causes of interest in using these technologies to the fashion and garment industry. When faster and accurate are the modeling studies, aesthetics and design of the products, these more competitive they become. Comparative purposes the tests were conducted with three male models and three female models covered in three height percentiles (5, 50 and 95\%) aged between 18 and 34 years, where the same pattern applied in the six models, comparing the behavior of the same in each.
\end{abstract}

Keywords: Three-dimensional scanning, Anthropometry, Surface Design.

\section{INTRODUÇÃO}

Já se sabe que na indústria têxtil a inovação é um fator essencial quando se desenvolve um produto, seja na criatividade, na produtividade, na tecnologia ou na personalização. Assim, a proposta deste estudo é analisar o tamanho mais favorável de estampas localizadas através da digitalização tridimensional a partir de medidas antropométricas baseada nos percentis de estatura.

A digitalização tridimensional é normalmente utilizada na captura de imagens e dados em 3D, que permitem extrair detalhes de superfícies, texturas e até objetos inteiros com grande precisão, através do auxílio de ferramentas computacionais (SILVA, 2006). A utilização dessas ferramentas digitais para o design têxtil, de acordo com Jones (1997), teve início na década de 1980, sendo vendidos como sistemas autônomos e conhecidos como CAD/CAM. Panero (2011) define a antropometria como a ciência que trata das medidas do corpo humano para determinar diferenças em indivíduos e grupos.

Segundo Silveira et. al (2007) a antropometria é uma ferramenta muito importante para os designers no desenvolvimento de produtos focado no usuário, visto que ao adquirir um produto ou serviço, o consumidor adquire um conjunto de benefícios que atendem suas expectativas.

\section{COMPORTAMENTO DO CONSUMIDOR}


A influência que as emoções geram em cima de nossas escolhas começou a ser documentada a partir dos trabalhos de Damásio (1996), neurocientista português que uniu pesquisa neurobiológica e investigação psicológica a fim de aprofundar os estudos sobre comportamento humano e as áreas cerebrais responsáveis pela tomada de decisão. De acordo com Love (2003), esses aspectos podem ser transportados ao design, auxiliando no processo de entendimento das interações entre homem e objeto. Kumar e Noble (2008) afirmam que o design pode sim ser a base para responder o que toda empresa busca saber sobre seu consumidor. Eles afirmam que a geração de emoções positivas, como prazer e fascinação, podem ser estimuladas através do produto pensado pelo design.

Para Lima e Kominsky (2010) as roupas são testemunhas de nossas vivências e estão incrustadas de mensagens significativas para nós. Por estarem em contato constante com a pele, precisam ser pensadas de forma "afetuosa" para que o consumidor se sinta especial, se sinta bem e satisfeito. A emoção é a primeira reação quando entramos em contato com o objeto, uma reação instantânea aos estímulos que recebemos.

Através da cor, das estampas, das texturas, acabamentos, modelagens, etc. os autores afirmam que se pode despertar sentimento de nostalgia nas pessoas, estreitando ainda mais os vínculos com a peça, aumentando as chances de o consumidor adquiri-la. Ao despertar lembranças de algo significativo, a pessoa acaba fazendo associações de emoções, chegando a crer que esta nova peça lhe fará se sentir da mesma forma que a anterior, fazendo uma correlação afetiva.

Um projeto de moda se torna relevante à medida que o consumidor valoriza e tem a necessidade de se sentir especial através da roupa que veste (LIMA; KOMINSKY, 2010). A partir desta necessidade, o consumidor acaba por buscar um conceito de vestuário mais focado no indivíduo, como se fosse projetado especialmente pra ele. Por isso cabe ao designer pensar em formas de estimular esse cliente, seja pela superfície trabalhada do tecido, seja pelos acabamentos ou pelas cores e formas que emprega em seu projeto, bem como os aspectos ergonômicos.

\section{DIGITALIZAÇÃO TRIDIMENSIONAL PARA OBTENÇÃO DE DADOS ANTROPOMÉTRICOS}

A antropologia é uma ciência cuja função é determinar as diferenças em indivíduos e grupos a partir das medidas corporais (PANERO, 2011). Inicialmente esse estudo era realizado tomando de forma superficial as medidas do corpo humano, como circunferência e largura utilizando instrumentos simples como fitas métricas, pinças e outros instrumentos de medição manual (JONES; RIOUXB, 1997). Conforme Panero (2011), a antropometria vai além de apenas tirar medidas, envolve variações de idade, gênero, etnia, fatores socioeconômicos e profissão. lida (2005) corrobora que é necessário analisar as particularidades de cada etnia, visto que a variação anatômica do corpo humano é bastante significativa, como mostra a figura 1. 


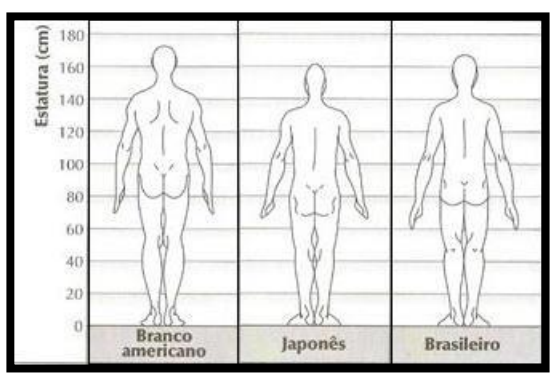

Figura 1: Variação de estatura em diferentes etnias.

Fonte: Adaptado de lida, 2005.

Os dados antropométricos podem ser expressos em percentis, que indicam a porcentagem de indivíduos com uma dimensão corporal de determinado tamanho. Panero (2011) atribui que para fins de estudo a população é dividida em 100 categorias percentuais da maior para a menor em relação a algum tipo especifico de medida corporal. O primeiro percentil de estatura ou altura, por exemplo, indica que $99 \%$ da população estudada teriam alturas maiores. Da mesma forma o percentil 95 indicaria que somente $5 \%$ da população estudada teriam alturas maiores e que $95 \%$ dessa população teriam a mesma altura ou menores.

A tabela 1 abaixo mostra os percentis 5, 50 e 95 de estatura de acordo com Panero (2011).

Tabela 1: Percentis de estatura

\begin{tabular}{|c|c|c|c|c|}
\hline \multicolumn{2}{|r|}{ IDADE } & Idade: 18 a 24 & Idade: 25 a 34 & Idade: 35 a 45 \\
\hline \multicolumn{2}{|r|}{ MEDIDA } & $\mathrm{cm}$ & $\mathrm{cm}$ & $\mathrm{cm}$ \\
\hline$\%$ & GÊNERO & & & \\
\hline \multirow{2}{*}{99} & HOMENS & 190,0 & 193,0 & 188,2 \\
\hline & MULHERES & 176,0 & 175,3 & 175,3 \\
\hline \multirow{2}{*}{95} & HOMENS & 185,7 & 187,5 & 184,2 \\
\hline & MULHERES & 172,5 & 170,9 & 170,7 \\
\hline \multirow{2}{*}{70} & HOMENS & 178,1 & 179,1 & 177,8 \\
\hline & MULHERES & 165,1 & 164,8 & 164,3 \\
\hline \multirow{2}{*}{50} & HOMENS & 174,2 & 175,3 & 174,2 \\
\hline & MULHERES & 162,3 & 161,8 & 161,0 \\
\hline \multirow{2}{*}{30} & HOMENS & 170,4 & 172,0 & 170,9 \\
\hline & MULHERES & 158,2 & 158,5 & 158,0 \\
\hline \multirow{2}{*}{5} & HOMENS & 163,3 & 163,6 & 163,1 \\
\hline & MULHERES & 152,4 & 151,6 & 151,4 \\
\hline
\end{tabular}




\begin{tabular}{c|c|c|c|c}
\hline \multirow{2}{*}{1} & HOMENS & 159,0 & 159,0 & 158,2 \\
\cline { 2 - 5 } & \multirow{2}{*}{ MULHERES } & 148,3 & 147,3 & 146,3 \\
\hline
\end{tabular}

Estatura* de adultos, sexo masculino e feminino, em centímetros $(\mathrm{cm})$ por idade, sexo e percentis selecionados**

*Altura sem sapatos. **Medida abaixo da qual recai a percentagem indicada de pessoas no respectivo grupo de idade.

Fonte: Adaptada de Panero (2011).

O estudo do corpo humano como um objeto 3D, de acordo com Jones e Riouxb (1997) nos permitiu estender o estudo das medidas corporais para a geometria e morfologia, principalmente externo humano de tecidos do corpo. A mesma inclui a aquisição, indexação, transmissão, arquivamento, recuperação, interrogatórios e análise de tamanho de corpo, forma e superfície.

O aprimoramento da antropometria se deu com os sistemas de digitalização do corpo humano, que existem a mais de quinze anos. Uma das principais usuárias dessa tecnologia com aplicação na área têxtil é a indústria militar, que a utiliza principalmente para otimizar o tempo de seleção de tamanhos dos uniformes para cada pessoa até possíveis assentos personalizados em aviões. Sendo que um dos grandes usuários da digitalização do corpo humano é a indústria cinematográfica, que utiliza desse recurso para efeitos especiais, simulações e dublagens (D'Apuzzo, 2007).

Segundo D’Apuzzo (2006) nas últimas décadas, os processos de controle dimensional passaram a utilizar máquinas de medição de coordenadas, onde podia-se obter uma grande precisão de medidas. No entanto, esta tecnologia também tem seus inconvenientes, pois a peça analisada deveria ficar fixa a uma mesa de medição.

\subsection{Modelos tridimensionais com aplicação na moda e indústria têxtil}

Atualmente, um dos maiores negócios do mercado é a indústria da moda. Esse fato deve-se não só ao alto volume de vendas e a sucessão de tendências em curto espaço de tempo, mas também à quantidade de pessoas e empresas envolvidas desde a concepção dos produtos até sua distribuição. Esses negócios têm sido pautados no aumento da produtividade e na oferta, cada vez mais rápida, para atender a demanda do consumidor (FEGHALI; DWYER, 2004). Por esse motivo existe um interesse especial em explorar a digitalização do corpo humano e aplicá-la a moda e a indústria de vestuário. A moda essencialmente envolve mudança, definida pela sucessão de tendências e manias em espaço curto de tempo, é um processo de obsolescência planejada (MIRANDA, 2008).

Os campos de atuação das tecnologias 3D são de natureza diversas, dentre elas: laser-scanning, projeção de padrões de luz (luz branca), a combinação de modelagem com transformação de imagem, baseadas em ativação de sensores, entre outras. $O$ escaneamento por laser e por luz branca são sistemas sem contato com o objeto a ser escaneado, baseados na emissão de radiação, onde um feixe pontual de luz (laser) ou um conjunto de feixes (luz branca) faz a varredura do objeto. O escaneamento por laser é mais preciso, porém por exigir uma posição estática da pessoa pode levar mais tempo 
para ser concluído. Já o escaneamento por luz branca é mais rápido, pois permite a projeção de várias linhas ao mesmo tempo. A combinação de modelagem com transformação de imagem (fotogrametria) é um sistema baseado na produção de um modelo 3D a partir de fotografias. A qualidade do modelo dependerá da resolução da câmera fotográfica utilizada, bem como a distância em que a foto foi tirada (SILVA et.al, 2010).

Segundo D'Apuzzo (2007) no âmbito comercial diferentes projetos de pesquisa foram realizados e já é possível encontrar scanners de corpo inteiro em shoppings centers e lojas. As tecnologias de digitalização 3D são aplicadas a diferentes partes do corpo humano e sistemas estão comercialmente disponíveis para a medição de praticamente qualquer área da superfície do corpo humano.

A tecnologia baseada na ativação de sensores utilizada em shoppings centers e lojas é baseada em imagem holográfica cilíndrica no corpo humano, onde o scanner faz uma varredura de todo o corpo enquanto com a pessoa totalmente vestida, emitindo ondas de rádio não-prejudiciais, de alta frequência para obter medições precisas do corpo. Todo o processo de digitalização dura cerca de 10 segundos e os dados coletados consistem em cerca de 200.000 pontos. Fora as medições, produz algoritmos automáticos que determinam cerca de 200 tamanhos de corpo característico com uma precisão de cerca de $6 \mathrm{~mm}$. Essa tecnologia auxilia o comprador a encontrar peças do vestuário que se adequem melhor ao seu biótipo corporal, bem como os tamanhos mais apropriados, a partir das medidas fornecidas pelo escaneamento (D'APUZZO, 2009).

\section{MÉTODOS E TÉCNICAS}

Este estudo foi realizado com o objetivo analisar o tamanho mais favorável de estampas localizadas através da digitalização tridimensional a partir de medidas antropométricas, baseadas nos percentis de estatura. Para parâmetros de comparação optou-se trabalhar com três percentis de estatura de 5\%, 50\% e $95 \%$ em homens e mulheres entre 18 e 34 anos, conforme foi tratado na seção 3 deste artigo.

Para obter os modelos tridimensionais do corpo humano desses percentis utilizou-se a digitalização tridimensional de luz branca. De acordo com D’Apuzzo (2009) a segunda tecnologia tridimensional amplamente utilizada para a medição do corpo humano baseia-se na projeção de padrões de luz. Em contraste com a varredura a laser, em vez de mover uma linha de laser único (ou vários) sobre o corpo humano, um padrão de luz, geralmente em forma de listras, é projetado no corpo humano. O dispositivo de digitalização é composto normalmente de um único projetor de luz branca e uma única câmera, que funciona de forma semelhante ao de uma câmera de captura de vídeo, que captura os dados digitalizados da superfície do objeto.

O modelo utilizado na pesquisa foi o digitalizador Artec Eva 3D scanner. Este scanner possui uma varredura rápida, precisa e oferece um modelo final com alta qualidade. Não requer marcadores ou calibração e captura objetos rapidamente em alta resolução e cores vibrantes.

Segundo o fabricante do Scanner Artec ${ }^{\mathrm{TM}}$ Eva é semelhante a uma câmera de vídeo que captura em 3D até 16 quadros por segundo. Estes quadros são alinhados automaticamente em tempo real, formando o modelo digital na tela do computador, conforme mostra a Figura 2. 

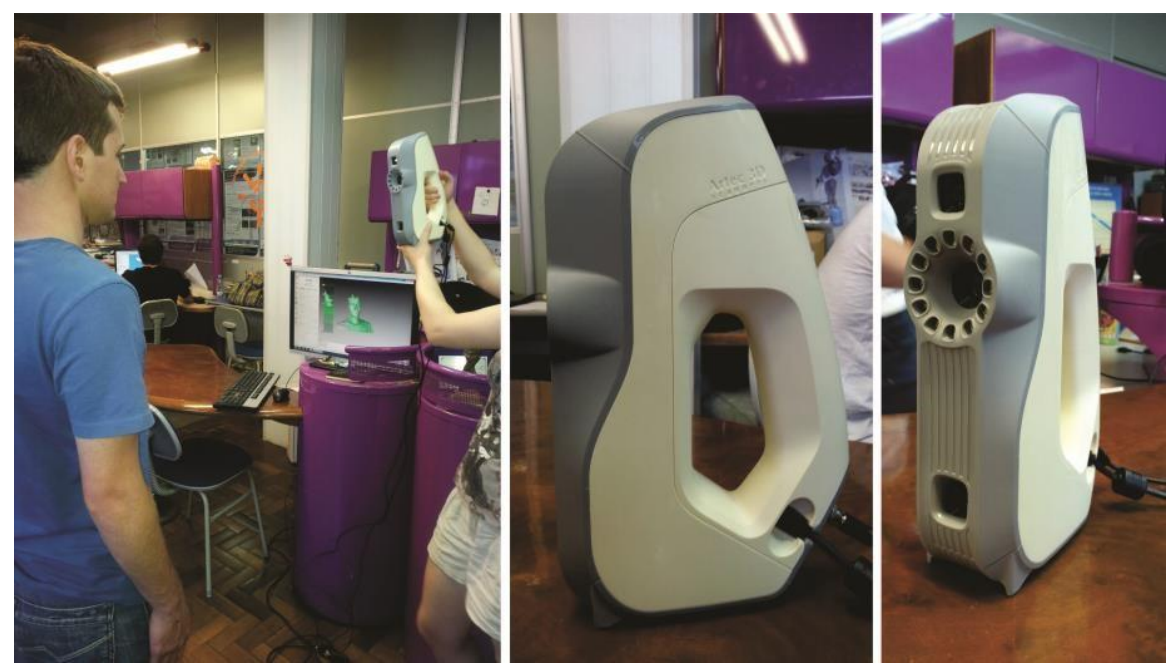

Figura 2: Digitalização com Artec Eva 3D scanner.

Fonte: Elaborado pelos autores.

Após a digitalização dos seis modelos humanos escolhidos a partir dos percentis especificados na seção 3, os arquivos gerados foram processados em um software específico de manipulação (software Raindrop Geomagic Studio ${ }^{\circledR} 10$ ). Nesta etapa, ocorreu a manipulação desses arquivos, a fim de se ter uma malha de pontos homogênea, sem espaços vazios, e a delimitação da área de trabalho a ser utilizada, onde manteve-se as dimensões e volumes dos modelos (Figura 3). O arquivo foi exportado em formato STL (Stereolitography) para poder ser transferido para outro software e ser aplicado a estampa.

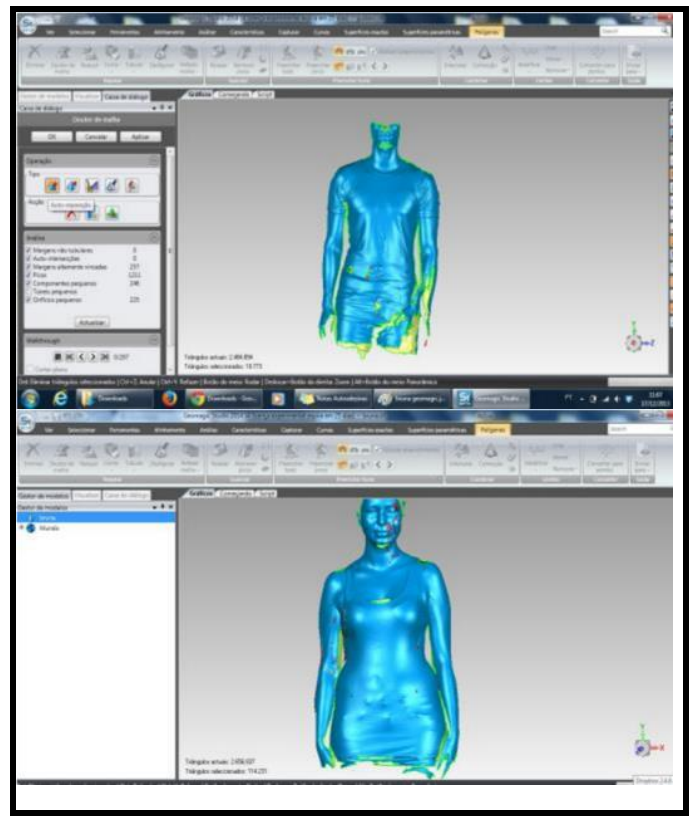

Figura 3: Manipulação no software Geomagic.

Fonte: Elaborado pelos autores.

$\mathrm{O}$ arquivo salvo em formato STL foi importado no software Keyshot ${ }^{\circledR}$, conforme $^{\circ}$ mostra a Figura 4. Para a realização da aplicação de uma estampa localizada, com tamanho de bastidor A4 nos modelos, a fim de se ter um modelo digital mais verossímil. 


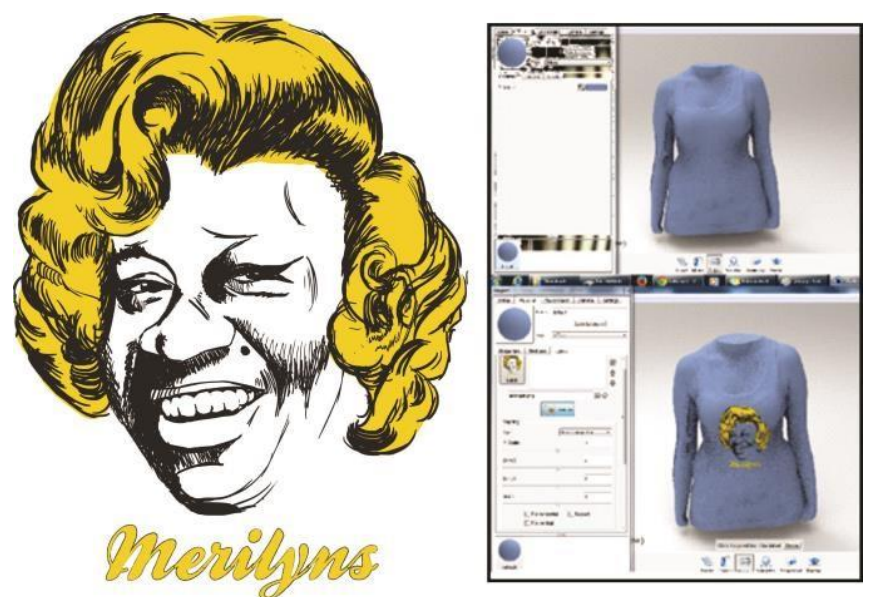

Figura 4: Estampa localizada utilizada na pesquisa e Manipulação modelos software Keyshot. Fonte: Elaborado pelos autores.

A estampa foi aplicada na dimensão padrão de $210 \times 297 \mathrm{~mm}$ nos três percentis, simulando a prática usual das empresas que prestam serviço de estampagem, possibilitando a verificação da falta de proporção da estampa, com a variação de tamanho do vestuário, objeto de estudo deste artigo.

Nas medições dos modelos digitais foram consideradas: a altura do tórax (ombro até o quadril), largura do tórax (peito) e circunferência do tórax conforme a Tabela 2.

Tabela 2: Medidas modelos digitais

\begin{tabular}{c|c|c|c|c} 
Modelo & Altura & Largura & Circunferência do tórax & Percentil \\
\hline Feminino 1 & $42,2 \mathrm{~cm}$ & $24,5 \mathrm{~cm}$ & $76,93 \mathrm{~cm}$ & $5 \%$ \\
\hline Feminino 2 & $46,2 \mathrm{~cm}$ & $28,2 \mathrm{~cm}$ & $88,54 \mathrm{~cm}$ & $50 \%$ \\
\hline Feminino 3 & $54,3 \mathrm{~cm}$ & $29,5 \mathrm{~cm}$ & $93,63 \mathrm{~cm}$ & $95 \%$ \\
\hline Masculino 1 & $48 \mathrm{~cm}$ & $30,6 \mathrm{~cm}$ & $96,08 \mathrm{~cm}$ & $5 \%$ \\
\hline Masculino 2 & $51,4 \mathrm{~cm}$ & $32,1 \mathrm{~cm}$ & $100,7 \mathrm{~cm}$ & $50 \%$ \\
\hline Masculino 3 & $63 \mathrm{~cm}$ & $37,9 \mathrm{~cm}$ & $119,06 \mathrm{~cm}$ & $95 \%$
\end{tabular}

Fonte: Elaborado pelos autores

A partir da circunferência do tórax é possível verificar nas grades de tamanho de vestuário já existentes qual o tamanho adequado a cada percentil analisado, tornando mais fácil e viável a aplicação do modelo de proporção de estamparia localizada proposto neste artigo.

Para a grade de tamanho masculina, foi utilizada a tabela: Referenciais de medidas do corpo humano - Vestibilidade para homens de tamanhos de corpo tipo normal, atlético e especial, produzida pela ABNT (Associação Brasileira de Normas Técnicas), disponível pela ABRAVEST (Associação Brasileira do Vestuário). Assim, os três 
modelos masculinos analisados (5\%, 50\% e 95\%) foram enquadrados nos tamanhos P, G e GG respectivamente.

Para a grade de tamanho feminina, não existe um modelo padrão, como o masculino. Cada empresa possui a sua padronagem, dificultando infinitamente a definição de tamanhos a partir da circunferência do tórax. Entretanto, muitos periódicos de moda indicam o livro Modelagem Industrial Brasileira como direção para criação de vestuário como referência. Assim, os três modelos femininos analisados enquadrados nos percentis antropométricos de 5\%, 50\% e 95\% foram enquadrados nos tamanhos PP, $\mathrm{M}$ ou $\mathrm{G}$ respectivamente.

\section{RESULTADOS E DISCUSSÕES}

A Figura 5 apresenta os modelos finais da aplicação das estampas localizadas nos indivíduos que se enquadram nos percentis de 5\%, 50\% e 95\% dos padrões antropométricos de estatura. Com isso, pode-se perceber a deficiência de um mesmo padrão de estampa em diferentes modelagens corporais.

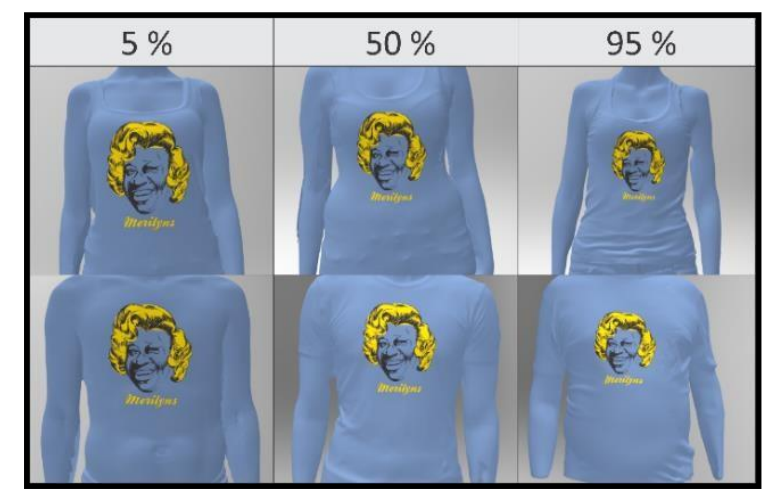

Figura 5: Aplicação estampa localizada nos modelos tridimensionais

Fonte: Elaborado pelos autores

O fato de o arquivo apresentar-se na forma digital manipulável em ambiente CAD (Computer-aided Design) apresenta a vantagem de permitir a comunicação visual entre o que se pretende produzir e as medidas reais do consumidor. Através da digitalização 3D existe a possibilidade de produzir peças personalizadas provindas de alta tecnologia geraria competitividade e exclusividade no mercado. Normalmente as estampas localizadas são produzidas em larga escala e utilizam um mesmo quadro para todos os tamanhos de camiseta a serem confeccionadas. Assim o risco de prejudicar a ideia base de uma estampa localizada é maior.

Sendo assim, foram calculadas as áreas totais de cada modelo digitalizado, simulando a área total de tecido da parte da frente de uma camiseta, sem considerar as mangas, o decote e a cava.

Calculando a área total de tecido usado, é possível comparar com a área total de um bastidor no tamanho A4, na posição vertical, verificando qual o espaço real e a porcentagem utilizada por uma estampa neste formato. Este resultado foi considerado como "percentual ideal para estampagem", onde o percentil de estatura de $50 \%$ foi utilizado como referência de proporção do tamanho ideal de estampa localizada. 0 tamanho do bastidor foi definido por ser uma medida padrão muito utilizada por 
empresas que prestam este serviço, além de representar uma dimensão adequada ao estudo deste artigo.

Como as medidas das alturas e larguras dos modelos feminino e masculino são diferentes, o percentual ideal para estampagem se mostrou diferente. Assim, verificouse que no modelo feminino o percentual ideal para estampagem foi de $45 \%$ da área do tecido e do masculino de $37,8 \%$.

Por conseguinte, fez-se o mesmo cálculo nos outros tamanhos para verificar qual o percentual de espaço um bastidor A4 ocuparia em tamanhos menores ou maiores de área de tecido. Os tamanhos pertencentes aos percentil $5 \%$ tiveram áreas superiores aos $45 \%$ e $37,8 \%$ verificados como ideal, bem como o percentil $95 \%$, que tiveram áreas inferiores, sendo necessário explorar um tamanho maior de bastidor.

Portanto, após calcular o percentual ideal de cada modelo, tendo como referência um bastidor a A4, foi possível verificar na Tabela 3 os resultados obtidos.

Tabela 3: Percentuais ideais de estampagem

\begin{tabular}{c|c|c|c} 
MODELO & PERCENTIL & TAMANHO COMERCIAL & $\begin{array}{c}\text { PERCENTUAL IDEAL DE } \\
\text { ESTAMPAGEM }\end{array}$ \\
\hline Feminino 1 & $5 \%$ & PP & $74 \%$ \\
\hline Feminino 2 & $50 \%$ & M & $100 \%$ \\
\hline Feminino 3 & $95 \%$ & G & $\begin{array}{c}115 \% \\
\text { (A4 não seria } \\
\text { suficiente) }\end{array}$ \\
\hline Masculino 1 & $5 \%$ & M & $84 \%$ \\
\hline Masculino 2 & $50 \%$ & G & $100 \%$ \\
\hline Masculino 3 & $95 \%$ & GG & $\begin{array}{c}144 \% \\
\text { (A4 não seria } \\
\text { suficiente) }\end{array}$ \\
\hline
\end{tabular}

Fonte: Elaborado pelos autores

Após, fez então a simulação dos tamanhos, conforme a proporção sugerida (Figura 6).

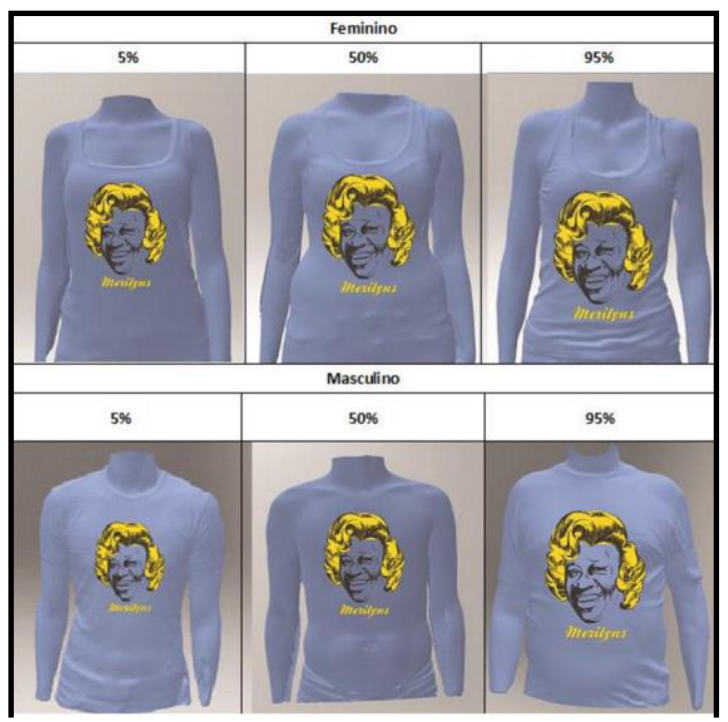

Figura 6: Simulação do percentual ideal de estampagem Fonte:

Elaborado pelos autores 


\section{CONCLUSÕES}

A partir das medidas antropométricas obtidas através da digitalização 3D, foi proposta nesta pesquisa, a análise da dimensão mais favorável para aplicação de estampas localizadas em camisetas personalizadas. Desta forma, percebeu-se que é possível manipular estampas de forma verossímil em modelos humanos virtuais bem como obter medidas antropométricas em software de CAD a partir de escaneamento do corpo real.

A base de dados permitiu a representação exata das estampas na camiseta que, podem ainda ser modificadas de acordo com as exigências do usuário. Assim, estabelece-se maior interação entre o designer/produtor e o consumidor final, que pode dispor de um produto totalmente adaptado ao seu gosto e estrutura corporal.

Verificou-se o grande potencial de utilização do Scanner Tridimensional de luz branca para o projeto de estampas localizadas e sua contribuição para o Design de artigos de moda e a possibilidade de produção de peças adequadas aos diferentes tamanhos.

Empresas que produzem artigos com estampas localizadas podem utilizar a técnica para calcular o tamanho ideal do desenho, mesmo que a variação respeitada seja a da peça de vestuário. Desta forma, as dimensões da estampa não ficam apenas a cargo da percepção estética e subjetiva do profissional de criação e quando bem explorada, pode ser um diferencial para o consumidor que busca produtos especiais, pensados exatamente para ele, estando disposto a pagar por isso.

Técnicas como a do escaneamento digital e de manipulação de malhas em 3D exigem algum tipo de conhecimento básico prévio, mas se mostram como uma excelente ferramenta para simulações e analises do corpo humano em tamanho real. Técnicas como a fotografia, podem tornar mais complicadas e demoradas as medições, já que podem não manter as proporções reais.

Estudos sobre grade padrão de tamanho do vestuário devem ser incentivadas, visto que existe disponível hoje apenas uma tabela de medidas padrão realizada pela $A B N T$, referente ao vestuário masculino, e nenhuma referente ao vestuário feminino e infantil, ficando a critério da marca a criação da própria grade de tamanhos.

\section{REFERÊNCIAS}

ABRAVEST. Disponível na internet por http em:

<http://www.abravest.org.br/index.php?option=com content\&view=article\&id=54\&lt emid=60>. Acesso em 26 de fev. 2014.

ARTEC3D. Disponível na internet por http em:

<http://www.artec3d.com/hardware/artec-eva/>. Acesso em 26 de fev. 2014.

DAMÁSIO, Antonio R. $O$ erro de Descartes: emoção, razão e o cérebro humano. São Paulo, Companhia das letras, 1996.

D'APUZZO, Nicola. 3D body scanning technology for fashion and apparel industry. In: 2007, Beraldin, J., Remondino, F., Shortis, M.R. (Eds.), Videometrics IX,Proc. of SPIE-IS\&T Electronic Imaging, SPIE Vol. 6491, San Jose (CA), USA. 
D'APUZZO, Nicola. Recent advances in $\mathbf{3 d}$ full body scanning with applications to fashion and apparel. In: 2009, Gruen, A., Kahmen, H. (Eds.), Optical 3-D Measurement Techniques IX, Vienna, Austria.

D'APUZZO, Nicola. Overview of 3D surface digitization technologies in Europe.In: 2006, Corner B.D., Li P., Tocheri M. (Eds.), Three-Dimensional Image Capture and Applications VI, Proc. ofSPIE-IS\&T Electronic Imaging, SPIE Vol. 6056, San Jose (CA), USA.

FEGHALI, marta kasznar; DWYER, Daniela. As Engrenagens da Moda. Rio de Janeiro: Senac Rio, 2004, 160p.

IIDA, Í. Ergonomia projeto e produção. São Paulo: Edgar Blucher, 2005.

JONES ,Peter R. M.; RIOUXB, Marc. Three-dimensional Surface Anthropometry: Applications to the Human Body. Optics and Lasers in Engineering 28 (1997) 89-117.

KUMAR, Minu; NOBLE, Charles $\mathrm{H}$. Using product design strategically to create deeper consumer connections. Business Horizons, Volume 51, Issue 5, Pages 441-45, 2008.

LIMA, Amanda Galvão Cruz; KOSMINSKY, Doris. O design emocional de moda. 2010. Disponível na internet por http em:

$<$ http://www.cetiqt.senai.br/dcb/ead/redige/index.php/redige/article/view/39/127>. Acesso em 26 de fev. 2014.

LOVE, T. Design and Sense: implications of Damasio's neurological findings for design theory. Proceedings of science and technology of design, sense ans sensibility in technology - Linking tradition to innovation throught design. Lisboa, Portugal, 2003.

MIB (Modelagem Industrial Brasileira). Disponível em:http://www.modelagemmib.com/Acesso em: 26 de fev. 2014.

MIRANDA, Ana Paula de. Consumo de moda: a relação pessoa-objeto. São Paulo: Estação das Letras e Cores, 2008.

PANERO, Julius; Zelnil, Martin. Human dimension \& interior space. A source book of design reference standdards. Tradução: Anita R. di Marco. Watson-Guptil Publications, New Yourk. 1ed. 6imp., 2011.

SILVA, F.P. O Uso da Digitalização Tridimensional a laser no Desenvolvimento e Caracterização de Texturas Aplicadas ao Design de Produto. Dissertação (Mestrado em Engenharia), PPGEM, UFRGS. Porto Alegre, 2006.

SILVA, F.P. Usinagem de Espumas de Poliuretano e Digitalização Tridimensional para a Fabricação de Assentos Personalizados para Pessoas com Deficiência. Tese (Doutorado em Engenharia), PPGEM, UFRGS, Porto Alegre, 2011.

SILVEIRA, Icléia; Silva, Giorgio Gilwan. Medidas Antropométricas e o Projeto do Vestuário. 3ำ Coloquio de Moda, 2007. Disponível na internet por http em: $<$ http://coloquiomoda.hospedagemdesites.ws/anais/anais/3-Coloquiodemoda 2007/8 11.pdf>. Acesso em 26 de fev. 2014. 